Int. J. Morphol.,

33(1):285-290, 2015

\title{
Perfil Antropométrico y Condición Física de Jugadores Veteranos de Básquetbol
}

\author{
Anthropometric and Fitness Profile of Senior Basketball Players
}

\begin{abstract}
Pablo Antonio Valdés-Badilla***; Andrés Esteban Roberto Godoy-Cumillaf*; Tomás Nicolás Herrera-Valenzuela ${ }^{* * *, * * * * *}$ \& Rodrigo Ramírez-Campillo ${ }^{* * * * *, * * * * * * * *}$
\end{abstract}

VALDÉS-BADILLA, P. A.; GODOY-CUMILLAF, A. E. R.; HERRERA-VALENZUELA, T. N. \& RAMÍREZ-CAMPILLO, R. Perfil antropométrico y condición física de jugadores veteranos de básquetbol. Int. J. Morphol., 33(1):285-290, 2015.

RESUMEN: Los cambios demográficos y sociales están produciendo un incremento progresivo de las personas de más de 60 años a nivel mundial, situación que no es distinta en Chile, por lo que estudiar variables relacionadas con este grupo de la población se vuelve importante. En este sentido, la presente investigación tiene por objetivo conocer el perfil antropométrico y condición física de jugadores veteranos de basquetbol (JVB). El tipo de investigación contempla un diseño descriptivo, transversal, con un enfoque cuantitativo. La muestra seleccionada incluyó a 30 JVB (edad 66,7 66,8 años) pertenecientes a la liga de la Asociación de Básquetbol Sénior de la Región de la Araucanía, Chile. Las variables dependientes estudiadas corresponden a la composición corporal, somatotipo y condición física (fuerza, resistencia aeróbica, flexibilidad, agilidad y equilibrio dinámico). Los resultados indican que los JVB mostraron un componente endomórfico, mesomórfico y ectomórfico de 4,1, 6,1 y 1,0, respectivamente, clasificándolos como meso-endomorfos, con una masa muscular de 45\% y masa grasa de $24,5 \%$. La condición física de los JVB presentó un rendimiento igual (40\% de los JVB) o superior (40\% de los JVB) al esperado según edad y sexo. En conclusión, los JVB poseen un perfil antropométrico y condición física favorable de acuerdo a su edad y sexo, sugiriendo que la práctica sistemática de básquetbol permitiría impactar positivamente sobre la composición corporal y el rendimiento físico-funcional en adultos mayores, sin embargo se requiere de mayor investigación para poder afirmarlo.

PALABRAS CLAVE: Composición corporal; Somatotipo; Condición física; Adulto mayor; Básquetbol.

\section{INTRODUCCIÓN}

Los cambios demográficos y sociales están produciendo un incremento progresivo de la población de más de 60 años a nivel mundial. Estos cambios se han producido por los bajos niveles de natalidad y altos índices de esperanza de vida de la población, lo que proyecta un aumento sustancial de personas mayores. De este modo se espera que en 2050 existan casi 395 millones de personas con más de 80 años, es decir, cuatro veces lo que existe hoy, superando la población de niños menores de 14 años (García Gil, 2012).

En Chile las cifras no son distintas al resto de los países desarrollados o en vías de desarrollo, y evidencia un aumento de la población mayor en las últimas décadas. De acuerdo a los datos otorgados por el Instituto Nacional de Estadísticas de Chile (INE, 2008), existen 2,6 millones de personas mayores de 60 años, lo que constituye un $15 \%$ de la pobla- ción nacional, y en algunos sectores del país (i.e. Región de la Araucanía) se eleva hasta 17,4\% (165 mil personas) (INE).

Durante el envejecimiento se reduce el repertorio motor, junto a la lentitud de los reflejos y descenso del tono muscular en reposo, entre otros factores, provocando descoordinación y torpeza motriz (Barbosa et al., 2007). Si bien el envejecimiento es un proceso normal del ser humano, la práctica regular de ejercicio físico puede atenuar algunos efectos negativos de este sobre la salud y calidad de vida (García Gil). Por otro lado, la inmovilidad e inactividad es el mayor agravante del envejecimiento y la incapacidad, de tal forma que lo que deja de realizarse fruto del envejecimiento, pronto será imposible realizar (Moreno, 2005). Lamentablemente, Chile presenta una alta tasa de sedentarismo, alcanzando $82,5 \%$ en población adulta y $86,5 \%$ en adultos mayo-

\footnotetext{
Instituto de Estudios en Ciencias de la Educación, Facultad de Educación, Universidad Autónoma de Chile, Sede Temuco, Chile.

** Departamento de Educación Física, Facultad de Educación, Universidad Autónoma de Chile, Sede Temuco, Chile.

**** Facultad de Ciencias de la Actividad Física, Universidad San Sebastián, Santiago, Chile.

**** Laboratorio de Ciencias de la Actividad Física, el Deporte y la Salud, Universidad de Santiago de Chile, Santiago, Chile.

******* Department of Physical Activity Sciences, Universidad de Los Lagos, Osorno, Chile.

******* Department of Physical Education, Sport and Recreation, Universidad de La Frontera, Temuco, Chile.
} 
res, alcanzando valores de hasta 90\% en algunas regiones (i.e. Región de la Araucanía) (Universidad de Concepción, 2012).

Tanto el envejecimiento de la población, como el sedentarismo asociado a este, podrían tener un impacto negativo en diversos sectores de la sociedad en general, y en la población de adultos mayores en particular. En consecuencia, estudios relacionados a la actividad física y el envejecimiento se hacen cada vez más relevantes. Algunos autores han descrito la condición física de adultos mayores en Iberoamérica (Correa Bautista et al., 2011; Vidarte Claros et al., 2012) y también sus características antropométricas (Izquierdo et al., 2004). Comparados a población normativa, los adultos mayores deportistas poseen mejores indicadores de condición física y antropometría, sin embargo, hasta donde los autores sabemos, no se han reportado estudios referentes a condición física y antropometría en adultos mayores que practican básquetbol en forma regular. Por tanto, la presente investigación tiene por objetivo conocer el perfil antropométrico y condición física de jugadores veteranos de basquetbol (JVB).

\section{MATERIAL Y MÉTODO}

El tipo de investigación contempla un diseño no experimental, descriptivo, transversal, con un enfoque cuantitativo. La población estuvo constituida por todos los basquetbolistas $(n=68)$ pertenecientes a la Asociación de Basquetbol Senior de la Región de la Araucanía, Chile y los criterios de inclusión fueron: Varones mayores de 60 años, pertenecer a un club activo en la liga regional de básquetbol, participar de la competencia de manera regular, ser autovalente y firmar un consentimiento informado que autorice el uso de los datos. Fueron excluidos los sujetos que presentaron alguna enfermedad inhabilitante, aquellos que poseían lesiones musculoesqueleticas que impidieran su normal desempeño físico y quienes ostentaran contraindicaciones permanentes o temporales para realizar ejercicio físico. La muestra seleccionada incluyó a 30 jugadores con los criterios indicados, dentro de los cuales se encontraba la totalidad del equipo representativo mayor de 60 años de la región de la Araucanía que participó del Campeonato Nacional de Básquetbol Senior realizado en Valdivia (Chile) en Noviembre de 2013, donde obtuvieron el primer lugar del Campeonato. El estudio fue aprobado por el Comité de Ética de la Universidad Autónoma de Chile y desarrollado siguiendo lo expuesto en la Declaración de Helsinki.

Todas las evaluaciones se realizaron en el mes de Septiembre de 2014, durante el periodo competitivo de los jugadores, ocupando un recinto cerrado con piso de madera, una temperatura promedio de $16{ }^{\circ} \mathrm{C}$ y $50 \%$ de humedad relativa.
La composición corporal fue establecida según el método descrito por Kerr (1988) y para el somatotipo se utilizó el método de Heath \& Carter (1967). Las medidas fueron tomadas según el protocolo descrito por la ISAK (Marfell-Jones et al., 2006). El peso corporal fue evaluado con balanza digital (Scale-tronix, USA), la estatura con estadiómetro (Seca modelo 220, Alemania), los diámetros con antropómetros (Rosscraft, Canadá), los perímetros con cinta métrica (Sanny, Brasil) y los pliegues cutáneos con cáliper (Harpenden, Inglaterra). Los instrumentos tienen una precisión de $0,1 \mathrm{~kg}, 0,10 \mathrm{~cm}, 0,1 \mathrm{~mm}, 0,1 \mathrm{~mm}$ y 0,2 $\mathrm{mm}$, respectivamente.

La condición física fue determinada de acuerdo al protocolo de la batería de test Senior Fitness, previamente descrito y validado para personas autovalentes sin problemas de salud entre 60 y 94 años de edad, el cual permite una evaluación de gran fiabilidad y fácil aplicación, junto con ofrecer una base de datos normativos importante (Rikli \& Jones, 2001). El orden de aplicación de las pruebas contempladas en la batería fue: prueba de sentarse y levantarse de una silla (SL) para evaluar fuerza de tren inferior, contabilizando el número de repeticiones en 30 s. Prueba de flexiones del brazo (FB) para evaluar fuerza de tren superior, utilizando una mancuerna de $5 \mathrm{lbs}$ y contabilizando el número de repeticiones en 30 s. Prueba de dos minutos de marcha ( 2 min) para evaluar resistencia aeróbica, registrando el número de elevaciones de rodillas. Prueba de flexión del tronco en silla (FTr) para evaluar flexibilidad de tren inferior, medido en $\mathrm{cm}$. Prueba de juntar las manos tras la espalda (JM) para evaluar flexibilidad de tren superior, medido en $\mathrm{cm}$. Prueba de levantarse, caminar y volver a sentarse (LCS) para evaluar agilidad y equilibrio dinámico, rodeando un cono a 2,44 $\mathrm{m}$ y registrando el tiempo en segundos. Las pruebas fueron controladas por el equipo de investigadores y desarrolladas tras un calentamiento de 15 minutos en el que se efectuaron ejercicios de movilidad articular y trabajo aeróbico. Todas las evaluaciones se realizaron previa participación de los sujetos en los partidos programados de la liga.

En relación al análisis estadístico, se utilizó planilla Microsoft Excel versión 7.0 para el vaciado preliminar de datos y para el tratamiento estadístico se usó el programa computacional GraphPad Prism 5.0 (Graphpad Software, San Diego, CA, USA). Las variables fueron sometidas a la prueba de normalidad de Kolmogorov Smirnov y a un análisis descriptivo calculando la media, desviación estándar (DE), valor mínimo y valor máximo. También se realizó un análisis de correlación (Pearson) para las variables dependientes del estudio, estableciendo un $\alpha$ en $\mathrm{P}<0,05$. 


\section{RESULTADOS}

Los JVB mostraron un componente endomórfico, mesomórfico y ectomórfico de 4,1, 6,1 y 1,0, respectivamente, clasificándose como mesoendomorfos (Tabla I). La ubicación promedio de la muestra en la somatocarta, se puede apreciar en la Figura 1.

En referencia a la composición corporal, los JVB presentaron un $24,5 \%, 45 \%, 14 \%, 11 \%$ y $5 \%$ de masa adiposa, muscular, residual, ósea y epitelial, respectivamente (Tabla I).

Respecto a la condición física, los JVB mostraron un rendimiento de 18 repeticiones, 19 repeticiones, 108 repeticiones, $3 \mathrm{~cm},-6 \mathrm{~cm}$ y $3,4 \mathrm{~s}$ en las pruebas de SL, FB, $2 \mathrm{~min}$, FTr, JM, LCS, respectivamente.

Al comparar la condición física de los JVB con tablas normativas según edad y sexo (Rikli \& Jones) cabe destacar que en la prueba de LCS, $80 \%$ de los JVB lograron la categoría de "sobre lo normal", mientras que en la prueba de JM, $50 \%$ clasificó en la categoría "bajo lo normal" (Tabla II).
El análisis de correlación mostró una relación significativa entre el test FTr y porcentaje (\%) de masa adiposa $(r=-0,38 ; p<0,05)$, así como entre FTr y porcentaje de masa muscular $(r=0,55 ; p<0,01)$. Además se observó una correlación significativa entre porcentaje de masa muscular y la prueba LCS $(r=-0,42 ; p<0,05)$.

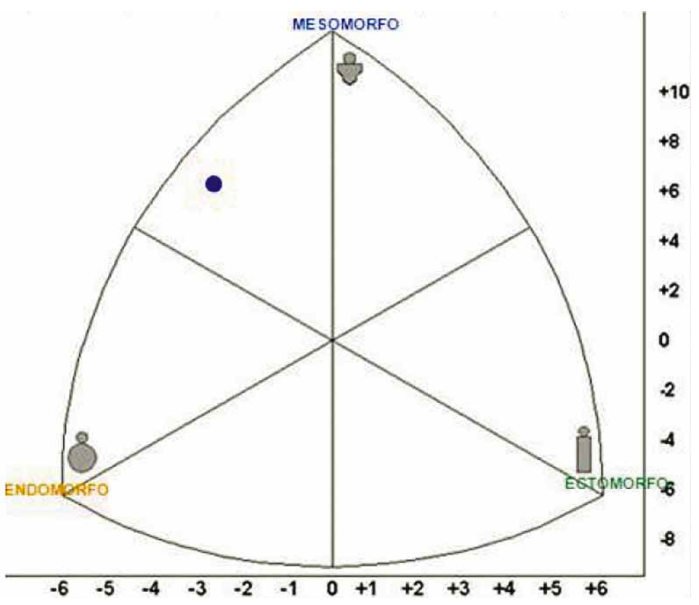

Fig. 1. Ubicación del somatotipo promedio de los jugadores veteranos de basquetbol en la somatocarta.

Tabla I. Características antropométricas de jugadores veteranos de basquetbol.

\begin{tabular}{lccc}
\hline Datos & Mínimo & Media \pm DE & Máximo \\
\hline Edad (años) & 60 & $66,7 \pm 6,8$ & 85 \\
ICC $(\mathrm{cm})$ & 0,91 & $0,96 \pm 0,03$ & 1,0 \\
Masa corporal (kg) & 62,1 & $83,9 \pm 12,1$ & 107,5 \\
Estatura (cm) & 157,2 & $173,9 \pm 9,1$ & 189,5 \\
IMC $\left(\mathrm{kg} / \mathrm{m}^{2}\right)$ & 20,8 & $27,7 \pm 2,8$ & 33,7 \\
Endomorfismo & 2,1 & $4,1 \pm 0,9$ & 5,9 \\
Mesomorfismo & 3,3 & $6,1 \pm 1,2$ & 8,1 \\
Ectomorf ismo & 0,1 & $1,0 \pm 0,7$ & 3,3 \\
Masa Adiposa (\%) & 19,0 & $24,5 \pm 3,0$ & 30,6 \\
Masa Muscular (\%) & 40,0 & $45,2 \pm 2,4$ & 49,7 \\
Masa Residual (\%) & 12,4 & $14,5 \pm 1$ & 16,5 \\
Masa Ósea (\%) & 9,2 & $11,2 \pm 1,1$ & 14,4 \\
Masa Cutánea (\%) & 4,1 & $4,6 \pm 0,4$ & 5,7 \\
\hline
\end{tabular}

ICC $=$ índice cintura/cadera; IMC $=$ índice de masa corporal.

Tabla II. Condición física de jugadores veteranos de basquetbol y clasificación normativa*.

\begin{tabular}{lcccccc}
\hline Prueba & Mínimo & Media \pm DE & Máximo & $\begin{array}{c}\text { Bajo lo } \\
\text { Normal- }\end{array}$ & Normal- & $\begin{array}{c}\text { Sobre lo } \\
\text { Normal_ }\end{array}$ \\
\hline SL (rep) & 12 & $17,9 \pm 4,1$ & 30 & $3,3 \%$ & $56,7 \%$ & $40 \%$ \\
FB (rep) & 12 & $19,4 \pm 3,2$ & 27 & $6,7 \%$ & $63,3 \%$ & $30 \%$ \\
2 min (rep) & 75 & $107,8 \pm 17,3$ & 148 & $3,3 \%$ & $70 \%$ & $26,7 \%$ \\
FTr (cm) & -21 & $3,3 \pm 9,7$ & 23 & $13,3 \%$ & $46,7 \%$ & $40 \%$ \\
JM (cm) & -27 & $-5,7 \pm 8,7$ & 13 & $53,4 \%$ & $23,3 \%$ & $23,3 \%$ \\
LCS (s) & 2,44 & $3,4 \pm 0,5$ & 5 & $16,7 \%$ & $0 \%$ & $83,3 \%$ \\
Promedio & --- & --- & --- & $20 \%$ & $40 \%$ & $40 \%$ \\
\hline
\end{tabular}

* = De acuerdo a Rikli \& Jones (2001). a= los valores representan un porcentaje del total de adultos mayores basquetbolistas; $\mathrm{SL}=$ Prueba de sentarse y levantarse de una silla; FB= Prueba de flexiones del brazo; 2 min= Prueba de 2 min de marcha; FTr= Prueba de flexión de tronco en silla; JM= Prueba de juntar las manos tras la espalda; LCS= Prueba de levantarse, caminar y volver a sentarse; Rep= Repeticiones. 


\section{DISCUSIÓN}

En lo que se refiere a la masa grasa, los JVB obtuvieron un $24,5 \%$, valor inferior al reportado previamente para sujetos sedentarios de la misma edad y sexo (Martínez Roldán et al., 2011). Esto podría ser explicado por el efecto de la actividad física (i.e. basquetbol) realizada por los JVB, la cual podría disminuir la grasa subcutánea en adultos mayores (García Gil), siendo la práctica regular un elemento clave para sustentar esta disminución (Izquierdo et al.).

A pesar que entre los 35 a 50 años de edad se produce una leve pérdida de masa muscular, la que luego se acelera hasta un 15\% por década (Young \& Skelton, 1994), la masa muscular de los JVB alcanzó un 45\%, lo cual los clasifica sobre valores normativos según edad y sexo (MarfellJones et al.). Si bien la genética podría jugar un rol en la masa muscular presentada por los JVB, es más probable que la práctica regular de basquetbol haya colaborado a que este grupo muestre menor atrofia muscular característica del envejecimiento, e incluso sea la responsable de estimular procesos hipertróficos (García Gil).

Los JVB presentaron una clasificación somatotípica mesoendomórfica, similar a la reportada en otros grupos de basquetbolistas, incluso de menor edad y alto nivel competitivo (Popovic et al., 2013). Respecto al endomorfismo, los JVB presentaron un valor de 4, superior al de basquetbolistas de menor edad (Popovic et al.), lo cual podría ser consecuencia de las diferencias en cuanto a frecuencia, volumen e intensidad de entrenamiento entre los dos grupos de deportistas. En relación a la mesomorfía, los JVB mostraron un valor de 6.1, superior al de basquetbolistas de menor edad (Popovic et al.), lo que podría explicarse por un alto perímetro de pantorrilla en los primeros. Referente a la ectomorfía, los JVB reflejaron un valor de 1 , inferior al reportado previamente para basquetbolistas de menor edad (Popovic et al.). Esta menor linealidad podría explicarse por el elevado componente mesomórfico e índice cintura/cadera (ver Tabla I) de los JVB.

En referencia a la condición física, se pudo observar que en la mayoría de las pruebas los JVB presentaron un rendimiento igual (40\% de los JVB) o superior ( $40 \%$ de los JVB) al esperado según edad y sexo. Esto concuerda con los hallazgos de estudios previos realizados con adultos mayores físicamente activos (Correa Bautista et al.; Vidarte Claros et al.), en donde se demostró el efecto positivo de la práctica regular de ejercicio físico sobre la fuerza de miembros inferiores y superiores (Lovell et al., 2010) sobre el equilibrio (Tiedermann et al., 2013) y sobre la resistencia aeróbica (Fleg, 2012), lo cual impactaría favorablemente sobre su independencia funcional (Young \& Skelton).
En cuanto a las pruebas de flexibilidad, los resultados indican buenos registros de los JVB para el miembro inferior, con $87 \%$ de los evaluados alcanzando valores de rendimiento normal o sobre lo normal. Por otro lado, un $53 \%$ de los JVB presentaron una flexibilidad de tren superior bajo lo normal. Esto podría explicarse por la importante masa muscular de los JVB (elevado perímetros de tórax y brazo), la cual podría limitar la flexibilidad de tren superior determinada por métodos indirectos (Dantas, 2003).

Para la prueba SL, los JVB obtuvieron 17,9 repeticiones, valor superior al observado en adultos mayores activos 13,9 (Milanovic et al., 2012). El mayor rendimiento de los JVB podría relacionarse con los estímulos intermitentes de alta intensidad encontrados en el basquetbol, lo que podría estimular la fuerza-potencia y un rendimiento muscular superior (Lovell et $a l$.). No obstante, existen investigaciones que han reportado mejores resultados para la misma prueba en mujeres (i.e. 20-25 repeticiones) (Pereira et al., 2010) sin embargo las mujeres fueron sometidas a entrenamiento de fuerza-potencia, incrementando su rendimiento hasta $60 \%$, adaptación neuromuscular significativa que podría ayudar a explicar la diferencia (Hernández \& García, 2013).

Respecto a la prueba de $2 \mathrm{~min}$, los JVB obtuvieron un promedio de 107,8 repeticiones, valor superior al observado en adultos mayores activos $(75,6)$ (Milanovic et al.). La mayor intensidad de ejercicio realizada por los JVB podría ayudar a explicar las diferencias (Lovell et al.).

En relación al test $\mathrm{FB}$, los $\mathrm{JVB}$ alcanzan un nivel de fuerza igual (60\%) o superior (30\%) al de personas de su misma edad y sexo. Escenario positivo, ya que la fuerza en adultos mayores se relaciona con mayor independencia en sus actividades cotidianas (Ensrud et al., 1994) y mejor calidad de vida (Benton et al., 2014).

En la prueba LCS los JVB presentaron un rendimiento de 3,4 s, superior al reportado previamente en adultos mayores que caminaban regularmente $(5,8-5,9 \mathrm{~s})$ (Milanovic et al.; Gouveia et al., 2013). Por tanto, en comparación a la caminata, los datos sugieren que la práctica regular de basquetbol podría estimular de manera más importante el equilibrio y la agilidad, permitiendo a adultos mayores un mejor rendimiento funcional en una actividad fundamental de la vida diaria como es el pararse, caminar y sentarse. 
Se observó relación entre las pruebas FTr y LCS con el porcentaje de masa muscular de los JVB, similar a lo reportado en otras investigaciones con personas mayores (Vidarte et al.; Ensrud et al.; Benton et al.), lo que se asocia a una mayor autonomía funcional y un menor riesgo de padecer caídas o lesiones relacionadas con las actividades de la vida cotidiana en adultos mayores (Vidarte Claros et al.; Milanovic et al.; Gouveia et al.).

\section{CONCLUSIÓN.}

En síntesis, los JVB poseen un perfil antropométrico y condición física favorable de acuerdo a su edad y sexo, sugiriendo que la práctica sistemática de básquetbol permitiría impactar positivamente sobre la composición corporal y el rendimiento físico-funcional en adultos mayores, sin embargo se requiere de mayor investigación para poder afirmarlo.

VALDÉS-BADILLA, P. A.; GODOY-CUMILLAF, A. E. R.; HERRERA-VALENZUELA, T. N. \& RAMÍREZ-CAMPILLO, R. Anthropometric and fitness profile of senior basketball players. Int. J. Morphol., 33(1):285-290, 2015.

SUMMARY: The demographic and social changes are causing a gradual increase in people over 60 years worldwide, situation thar is similar in Chile, so the study of variables related to this group of the population becomes important. In this regard, the aim of this study is to determine the anthropometric and physical fitness profile of senior basketball players (SBP). This is a descriptive, cross-sectional, quantitative research. The sample included $30 \mathrm{SBP}$ (age $66.7 \pm 6.8$ years) belonging to the league of the Senior Basketball Association of the Araucanía Region, Chile. The dependent variables studied correspond to body composition, somatotype and fitness (strength, endurance, flexibility, agility and dynamic balance). Results indicate that the SBP showed endomorphic, mesomorphic and ectomorphic values of 4.1, 6.1 and 1.0, respectively, as meso-endomorphs with a muscle mass of $45 \%$ and body fat of $24.5 \%$. Compared to age and sex counterparts, SBP exhibit equal (40\% of SBP) or even higher ( $40 \%$ of SBP) values for physical fitness perfomance variables. In conclusion, SBP have a favorable anthropometric and physical fitness profile for their age and sex, suggesting that the systematic practice of basketball would have a positive impact on body composition and physical fitness-function in older adults, however more experimental research is needed to corroborate this.

KEY WORDS: Body composition; Somatotype; Fitness; Elderly; Basketball.

\section{REFERENCIAS BIBLIOGRÁFICAS}

Barbosa, M. J. A. P.; Rodríguez, M. N. G.; Hernández, H. V. Y. M.; Hernández, H. R. A. \& Herrera, M. H. A. Masa muscular, fuerza muscular y otros componentes de funcionalidad en adultos mayores institucionalizados de la Gran Caracas-Venezuela. Nutr. Hosp., 22(5):578-83, 2007.

Benton, M. J.; Alexander, J. L. \& Holland, J. D. Relationship between strength, function, and quality of life in older adults with chronic lung disease: is there an influence of gender? $J$. Cardiopulm. Rehabil. Prev., 34(2):143-9, 2014.

Correa Bautista, J. E.; Gámez Martinez, E. R.; Ibáñez Pinilla, M. \& Rodríguez Daza, K. D. Aptitud física en mujeres adultas mayores vinculadas a un programa de envejecimiento activo. Salud U. I. S., 43(3):263-9, 2011.

Dantas, E. H. M. A prática da prepararação física. $5^{\text {a }}$ ed. Río de Janeiro, Shape, 2003.

Ensrud, K. E.; Nevitt, M. C.; Yunis, C.; Cauley, J. A.; Seeley, D. G.; Fox, K. M. \& Cummings, S. R. Correlates of impaired function in older women. J. Am. Geriatr. Soc., 42(5):481-9, 1994.

Fleg, J. L. Aerobic exercise in the elderly: a key to successful aging. Discov. Med., 13(70):223-8, 2012.
García Gil, M. Manual de ejercicio físico para personas de edad avanzada. Bizkaia, Dirección General de Deporte y Juventud de la Diputación Foral de Bizkaia, 2012.

Gouveia, É. R.; Maia, J. A.; Beunen, G. P.; Blimkie, C. J.; Fena, E. M. \& Freitas, D. L. Functional fitness and physical activity of Portuguese community-residing older adults. J. Aging Phys. Act., 21(1):1-19, 2013.

Heath, B. H. \& Carter, J. E. A modified somatotype method. Am. J. Phys. Anthropol., 27(1):57-74, 1967.

Hernández, Y. H. \& García, J. M. Efectos de un entrenamiento específico de potencia aplicado a futbolistas juveniles para la mejora de la velocidad con cambio de dirección. Mot. Eur. Jo. Hum. Mov., 31:17-36, 2013.

Instituto Nacional de Estadísticas (INE). Población y Sociedad: Aspectos Demográficos. Santiago, Instituto Nacional de Estadísticas (INE), 2008. Disponible en: http://www.ine.cl/canales/chile_estadistico/demografia_y_vitales/demografia/pdf/ poblacion_sociedad_enero09.pdf

Izquierdo, M.; Ibañez, J.; Hakkinen, K.; Kraemer, W. J.; Larrión, J. L. \& Gorostiaga, E. M. Once weekly combined resistance and cardiovascular training in healthy older men. Med. Sci. Sports Exerc., 36(3):435-43, 2004. 
Kerr, A. D. An anthropometric method for fractionation of skin, adipose, bone, muscle and residual tissue masses in males and females age 6 to 77 years. MsC. Kinesiology Tesis. Burnaby, Simon Fraser University, 1988.

Lovell, D. I.; Cuneo, R. \& Gass, G. C. The effect of strength training and short-term detraining on maximum force and the rate of force development of older men. Eur. J. Appl. Physiol., 109(3):429-35, 2010.

Marfell-Jones, M.; Olds, T.; Stewart, A. \& Carter, L. International Standards for Anthropometric Assessment. Potchefstroom, South Africa, International Society for the Advancement of Kinanthropometry (ISAK), 2006.

Martínez Roldán, C.; Veiga Herreros, P.; Cobo Sanz, J. M. \& Carbajal Azcona, A. Evaluación del estado nutricional de un grupo de adultos mayores de 50 años mediante parámetros dietéticos y de composición corporal. Nutr. Hosp., 26(5):108190, 2011.

Milanovic, Z.; Pantelic, S. \& Jorgic, B. Changes in physical fitness of men older than 60 - A pilot study. SportLogia, 8(1):43-9, 2012.

Moreno, G. A. Incidencia de la actividad física en el adulto mayor. Rev. Int. Med. Cienc. Act. Fis. Deporte, 5(19):222-37, 2005.

Pereira, A.; Izquierdo, M.; Silva, A. J.; Costa, A. M.; Bastos, E.; González-Badillo, J. J. \& Marques, M. C. Effects of high-speed power training on functional capacity and muscle performance in older women. Exp. Gerontol., 47(3):250-5, 2012.

Popovic, S.; Akpinar, S.; Jaksic, D.; Matic, R. \& Bjelica, D. Comparative study of anthropometric measurement and body composition between elite soccer and basketball players. Int J. Morphol., 31(2):461-7, 2013.

Rikli, R. \& Jones, C. Senior fitness test manual. Champaign (IL), Human Kinetics, 2001.

Tiedemann, A.; Sherrington, C. \& Lord, S. The role of exercise for fall prevention in older age. Mot. Rev. Educ. Fis., 19(3):5417, 2013.

Universidad de Concepción. Encuesta nacional de hábitos de actividad física y deportes en la población Chilena de 18 años y más. Concepción, Facultad de Ciencias Económicas y Administrativas, Universidad de Concepción, 2012. Disponible en: http://www.ind.cl/estudios-e-investigacion/investigaciones/ Documents/2012/Encuesta\%20Act\%20Fisica/encuesta-actfisica-2012.pdf

Vidarte Claros, J. A.; Quintero Cruz, M. V. \& Herazo Beltrán, Y. Efectos del ejercicio físico en la condición física funcional y la estabilidad en adultos mayores. Hacia Promoc. Salud, 17(2):79-90, 2012.
Young, A. \& Skelton, D. A. Applied physiology of strength and power in old age. Int. J. Sports Med., 15(3):149-51, 1994.

Dirección para Correspondencia:

Pablo Antonio Valdés Badilla

Universidad Autónoma de Chile

Porvenir 649

Temuco

CHILE

Email: pablo.valdes@uautonoma.cl

Recibido: 01-10-2014

Aceptado: 07-01-2015 